\title{
Noninvasive ventilation for acute respiratory failure: a prospective randomised placebo-controlled trial
}

\author{
F. Thys*, J. Roeseler\#, M. Reynaert*, G. Liistro", D.O. Rodenstein"
}

Noninvasive ventilation for acute respiratory failure: a prospective randomised placebocontrolled trial. F. Thys, J. Roeseler, M. Reynaert, G. Liistro, D.O. Rodenstein. (C) ERS Journals Ltd 2002.

ABSTRACT: The aim of the present study was to clarify whether the known effects of noninvasive positive-pressure ventilation (NPPV) in patients with respiratory failure are real or due to placebo effects and whether early application of NPPV in the emergency department leads to rapid improvement of the patients condition and outcome.

A prospective randomised placebo-controlled study was conducted in 20 patients with severe acute respiratory failure (ARF) secondary to an acute exacerbation of chronic obstructive pulmonary disease (COPD) or acute pulmonary oedema, not improving under conventional medical therapy and on the edge of intubation. Patients received either conventional medical therapy plus two-level NPPV (bi-level NPPV) or conventional medical therapy plus "placebo" NPPV.

The main outcome measures involved the need for endotracheal intubation in the bilevel NPPV arm and in the placebo arm after crossing over to active NPPV. Morbidity, length of stay, mortality and the effect of the ventilatory mode on clinical, arterialblood gas parameters, and the sternocleidomastoid muscles electromyogram (EMG) activity were also measured.

The 10 patients in the active NPPV group rapidly improved and none needed intubation. Placebo NPPV resulted in no change in the clinical condition of patients that continued to worsen and the 10 patients were crossed over to active NPPV. Three patients were intubated. No differences in terms of morbidity, length of stay or mortality between the two groups were observed. Active NPPV (but not placebo NPPV) led to a rapid and significant improvement in the clinical parameters, $\mathrm{pH}$ and the carbon dioxide tension in arterial blood and to a decrease in respiratory frequency and sternocleidomastoid EMG activity.

Early application of bi-level noninvasive positive-pressure ventilation in patients with severe acute respiratory failure, due to chronic obstructive pulmonary disease and acute pulmonary oedema, leads to a rapid improvement in clinical status and blood gases. Noninvasive positive-pressure ventilation had no placebo effect. Eur Respir J 2002; 20: 545-555.
Depts of *Emergency, $\quad{ }^{*}$ Physical Therapy and "Pneumology, Cliniques universitaires Saint-Luc, Université catholique de Louvain, Brussels, Belgium.

Correspondence: F. Thys

Service des Urgences

Cliniques universitaires Saint-Luc

Université catholique de Louvain

Avenue Hippocrate 10

B-1200 Bruxelles

Belgium

Fax: 3227641620

E-mail: Thys@rean.ucl.ac.be

Keywords: Acute respiratory failure emergency department noninvasive ventilation

Received: October 192001

Accepted after revision: March 192002

The present study was partly supported by a grant to $F$. Thys from the "Fondation Saint-Luc".
Patients with acute respiratory distress usually arrive at the hospital via the emergency department (ED), where the initial management leads quite rapidly either to improvement and subsequent transfer to a medical ward, or transfer into an intensive care unit (ICU). In extreme cases, patients unresponsive to medical therapy are submitted to mechanical ventilation. The latter is usually administered in the ICU rather than in the ED. Until recently, mechanical ventilation required endotracheal intubation. Currently, several well-conducted studies have shown that noninvasive positive-pressure ventilation (NPPV) via a nasal or facial mask is at least as effective as invasive ventilation in several conditions, with less complications and better outcomes [1-11].

Two problems remain to be answered. On the one hand, at least one study in patients with moderate-tosevere chronic obstructive pulmonary disease (COPD) in acute respiratory failure (ARF) has shown that
NPPV added no value to well-conducted medical treatment, casting some doubt on the real value of NPPV, at least in patients not needing immediate transfer to the ICU [12]. A second study found an increased rate of myocardial infarction in patients with respiratory failure due to acute pulmonary oedema (APO) treated with NPPV [13]. On the other hand, all studies proving the benefit of NPPV in this acute setting were performed either in the ICU or the general ward, that is some (unspecified) time after the initial admission of the patient via the ED. During this unspecified time, patients were probably treated with the usual medical therapy, including unknown amounts of oxygen $\left(\mathrm{O}_{2}\right)$ supplementation. Patients could have improved, deteriorated or remained stable during this time. Therefore, patients arriving in the wards or ICU are not necessarily in the same status as when they arrived in the ED. Early institution of noninvasive mechanical ventilation in 
the ED seems feasible, and some data suggest that it could lead to rapid improvement in some patients [14-17].

However, NPPV, by its very nature, could imply a powerful placebo effect leading to clinical improvement, for instance in dyspnoea, tachypnoea, anxiety and agitation, independent of the improvement due to medical treatment. It is impossible to separate these possible effects, since in all studies performed to date NPPV plus medical therapy was compared to medical therapy alone or intubation and mechanical ventilation. This is not a trivial matter, since many patients could be submitted to useless treatment in the ED, ICU and also the general wards. Indeed, presently, there is a generalised tendency to institute NPPV in many patients not necessarily representative of the patients included in the well-designed studies published in the literature.

This randomised, placebo-controlled single-blind study was designed to answer two questions: 1) can NPPV be performed in an ED very early after patient admission with a similar benefit seen in the need for intubation, length of stay and mortality as in previous studies and; 2) does early NPPV have a real, rather than a placebo effect, both on objectively measured parameters and on the clinical status of the patient. According to previous literature, only patients with two primary diagnoses (COPD and APO) were studied.

\section{Material and methods}

\section{Study location and patient selection}

The study protocol was approved by the Ethical Practices Committee of the authors' hospital in accordance with the principles of good clinical practice. Informed oral consent was obtained from all study participants. The study was conducted in the ED of an urban university teaching hospital (884 beds). During a 2-yr period (1999-2000), patients with acute respiratory distress secondary to either an acute exacerbation of COPD or APO were eligible for this investigation. Cardiogenic pulmonary oedema was defined as orthopnoea, bibasilar crackles, bilateral perihilar infiltrates on chest radiograph with cardiomegaly and a compatible clinical history. An acute exacerbation of COPD was defined as acute respiratory distress in a cigarette smoker with a known history of long-lasting dyspnoea on exertion with frequent exacerbations and cough, and mucus hyperproduction, without symptoms or signs of other specific causes (absence of pneumothorax, pneumonia, pleural effusion, no reason to suspect an episode of pulmonary embolism). Patients were entered into the study if they were aged $>18$ yrs and had evidence of ARF as demonstrated by three of the following criteria: acute onset of moderate-to-severe dyspnoea as assessed by the ED physician who took care of the patient; a respiratory rate $>30($ or $<10)$ breaths $\cdot \mathrm{min}^{-1}$; hypoxaemia (oxygen tension in arterial blood $\left(\mathrm{Pa}, \mathrm{O}_{2}\right)$ $<7.3 \mathrm{kPa}\left(55 \mathrm{mmHg}\right.$ ) (on room air)) or need for $\mathrm{O}_{2}$ supplementation; respiratory acidosis $(\mathrm{pH}<7.33)$. The diagnosis of ARF and the decision to include the patient into the study was the responsibility of the ED physician (independent of the investigators). Patients were excluded from this investigation if they had any of the following: 1) an immediate indication for endotracheal intubation (respiratory and/or cardiac arrest); 2) major unrest; 3) haemodynamic instability despite a fluid challenge; 4) facial or thoracic trauma; 5) lack of cooperation; 6) difficult adaptation of a facial mask to a patient's facial anatomy; 6) clinical suspicion of pulmonary embolism; 7) retrosternal pain suggestive of a myocardial ischaemia even with a normal admission electrocardiogram (ECG).

\section{Study design}

The trial was designed to enrol 60 patients (on the basis of previous studies, the percentage of patients expected to respond to conventional therapy at 30 and $70 \%$ NPPV was evaluated). Therefore, with 30 patients per group, there was a $95 \%$ chance of rejecting the null hypothesis [18]. Planned interim analysis was performed after 20 and 40 patients had been enrolled. Patients were eligible into the study if, after an initial period of medical treatment, the attending physician judged that intubation and mechanical ventilation was to be considered. The following initial treatments were administered: supplemental $\mathrm{O}_{2}$, intravenous vasodilators (isosorbide dinitrate $2 \mathrm{mg} \cdot \mathrm{h}^{-1}$ ) and intravenous diuretics (furosemide $40 \mathrm{mg}$ ) for cardiogenic pulmonary oedema and; supplemental $\mathrm{O}_{2}$, bronchodilatoraerosol therapy (fenoterol $1,500 \mu \mathrm{g}$ and ipratropium bromide $0.4 \mathrm{mg}$ ) repeated every $20 \mathrm{~min}$ and intravenous glucocorticoids (methylprednisolone $80 \mathrm{mg}$ ) for acute exacerbation of COPD. Patients not improving under this treatment were included into the study while this treatment continued. Patients were randomly assigned to receive conventional medical therapy plus two-level NPPV (bi-level NPPV) or conventional medical therapy plus "placebo" NPPV. Randomisation was performed using opaque, sealed envelopes which were opened at the time of inclusion into the study by batches of 20 envelopes. The study treatment was continued until the attending physician (who was continuously present throughout the study at the bedside) interrupted the treatment either after the patient improved or after the treatment was considered to have failed. Failure was defined in advance as a deterioration in clinical status including all of the following: dyspnoea, respiratory and/or heart frequency, sweating and agitation or deterioration in blood gases and/or in haemodynamic status. Success was defined as clear improvement in both the blood gases and the clinical status. Treatment success led to study end. In case of treatment failure, endotracheal intubation was applied to patients in the active NPPV group. Patients in the placebo group, in whom intubation was deemed necessary, were submitted first to active NPPV (rescue protocol). At all times the attending physician could decide to interrupt the study. 
Active bi-level noninvasive positive-pressure ventilation protocol

Patients randomised to receive bi-level NPPV were evaluated by two investigators. With respect to the time of arrival of the patient to the ED, this implied a delay dependent on two factors: the time necessary for the ED physician to decide inclusion and the time necessary for the investigators to arrive to the ED and start the protocol. Bi-level NPPV was delivered

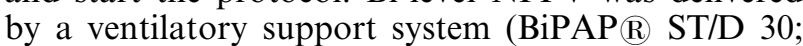
Respironics, Inc., Murrysville, PA, USA) with a standard expiratory port. Bi-level NPPV was instituted with a face mask (Bird, Bird Corp., CA, USA) with the patient in a semirecumbent position. The expiratory pressure was set at the minimal pressure level $\left(4 \mathrm{cmH}_{2} \mathrm{O}\right)$ and the inspiratory pressure was set at $10 \mathrm{cmH}_{2} \mathrm{O}$. The machine was used in the assistcontrol mode with a backup frequency of $10 \cdot \mathrm{min}^{-1}$. In all patients, the inspiratory pressure was increased by $2 \mathrm{~cm}$ of water steps, until the patient showed signs of discomfort (increasing sensation of dyspnoea) or leaks were observed between the face mask and the skin or a pressure of $20 \mathrm{cmH}_{2} \mathrm{O}$ was reached. Thereafter, expiratory pressure was similarly increased until discomfort appeared. A gastric tube was not used. During the institution of bi-level NPPV, $\mathrm{O}_{2}$ was added with a nasal catheter inside the mask as needed to obtain a saturation of $90 \%$ (assessed by pulse oxymetry).

\section{Placebo noninvasive positive-pressure ventilation protocol}

The design of the placebo NPPV protocol was strictly the same as the active bi-level NPPV protocol. Placebo-device ventilation was delivered by the same ventilatory support system. In this arm of the protocol, the T-connector piece of the device between the mask and the tubing was substituted by a specially designed T-connector with several holes. With this pierced $\mathrm{T}$-connector, the inspiratory and expiratory pressures delivered through the face mask were equal to zero. The influence of the placebo NPPV on the respiratory work of breathing (WOB) and its rebreathing (dead space) effect had been assessed previously in five and seven normal subjects respectively (see below) and were found to result in no significant change in WOB or increase in end-tidal carbon dioxide $\left(P\right.$ ET, $\left.\mathrm{CO}_{2}\right)$.

During the institution of this placebo-device ventilation, $\mathrm{O}_{2}$ was added with a nasal catheter inside the mask as needed to obtain a saturation of $90 \%$.

\section{Outcome measures}

The main outcome measure was the difference in the number of patients failing noninvasive ventilation in each arm of the study. For the active arm, failure was defined as the need for endotracheal intubation; for the placebo arm, failure was defined as the need to stop placebo NPPV and to cross over to active ventilation (either noninvasive ventilation or, if this failed, through endotracheal intubation). As secondary end points, the effects of the ventilatory mode on the clinical and arterial-blood gas parameters, and on sternocleidomastoid muscle activity were evaluated. Hospital mortality, admission to the ICU, the length of ED stay, the length of ICU stay and the length of hospital stay were also assessed.

\section{Physiological measurements}

During the clinical study, the intensity of dyspnoea was measured by a $10-\mathrm{cm}$ long visual analogue scale with 0 measuring normal breathing and 10 unbearable dyspnoea. Dyspnoea was assessed by the patient, and also by the nursing team and the investigators, at different times throughout the protocol (see below). The arterial blood gases $\left(\mathrm{pH}, P \mathrm{~Pa}, \mathrm{O}_{2}\right.$, carbon dioxide tension in arterial blood $\left(\mathrm{Pa}_{\mathrm{a}} \mathrm{CO}_{2}\right)$ and arterial oxygen saturation) were measured at the time of randomisation, after 20 min of active or sham ventilation, and at the end of ventilatory assistance. The following parameters were continuously recorded in digital form: ECG from three surface electrodes placed on the chest wall, pulse rate and pulse oxymetry from a Datex oxymeter (Datex, Helsinki, Finland) equipped with a finger probe, systolic and diastolic blood pressure with an arm-brassard sphygmomanometer (Datex), the thoracic and abdominal respiratory movements and their sum obtained with an uncalibrated respiratory inductive plethysmograph (Respitrace $\mathbb{R}$, Ambulatory Monitoring, Ardsley, NY, USA), the electromyogram (EMG) of the sternocleidomastoid activity obtained from surface electrodes placed upon the sternocleidomastoid muscles. All signals were digitised and recorded on a dedicated device, specially constructed for this study. In addition to the above signals, the

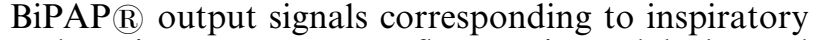
and expiratory pressures, flow, estimated leaks and tidal volume were also entered into the recording device.

The cardiac enzymes (creatine kinase (CPK), glutamic oxaloacetic transaminase (GOT), lactate dehydrogenase (LDH), Troponine) were also measured on inclusion into the study. The complications of bi-level NPPV (skin damage, gastric dilatation, vomiting) were recorded.

\section{Influence of placebo noninvasive positive-pressure ventilation protocol on the work of breathing and carbon dioxide rebreathing}

WOB was assessed in five normal volunteers by simultaneously recording the tidal volume with a calibrated inductive plethysmograph, and transpulmonary pressure with an oesophageal balloon positioned $40 \mathrm{~cm}$ from the nares. Measurements were performed during a 1-min stable-breathing period under three conditions: spontaneous breathing; breathing spontaneously but with the facial mask placed upon the face; spontaneous breathing with the placebo NPPV device. Transpulmonary pressure was 
plotted against tidal volume for all recorded breaths, and the mean WOB was calculated per litre of tidal volume.

The $\mathrm{CO}_{2}$ rebreathing effects of the placebo NPPV were measured in a similar way in another group of seven normal subjects, by recording the $P$ ET, $\mathrm{CO}_{2}$ using a hollow nasal sampling device (Datex $\mathrm{CO}_{2}$ analyser). Measurements were performed during a 1 min stablestate period during spontaneous breathing, application of the facial mask upon the face, and application of placebo NPPV.

\section{Statistical analysis}

Values are presented as mean \pm SD. T-tests for paired and unpaired samples were used to compare the variables. When more than two samples had to be compared, one-way analysis of variance (ANOVA) was used. One-way ANOVA with repeated data was used to compare the frequency of breathing. For WOB and $P$ ET, $\mathrm{CO}_{2}$ comparisons, the Wilcoxon-rank test was used.

\section{Results}

At the time of the first interim analysis of data from 20 patients, enrolment was suspended because the differences in the failure rate and clinical evolution between the two groups were clear cut enough.

\section{Patients}

During the study period, $\sim 1,300$ patients were admitted to the ED for acute respiratory distress. A total of 187 of these patients had a diagnosis of APO or acute exacerbation of COPD. The investigators were contacted for this study in 65 cases ( 37 acute exacerbations of COPD and 28 APO). Twenty patients $(30.7 \%)$ were enrolled into the study. Of the remaining 45 patients: seven $(10.8 \%)$ required immediate endotracheal intubation; eight $(12.2 \%)$ had chest pain or evidence of cardiac ischaemia; $11(16.9 \%)$ had no criteria for inclusion; seven $(10.8 \%)$ patients with COPD exacerbation had hypoxaemic pneumonia; eight $(12.2 \%)$ patients were not enrolled because the two investigators were not available; two (3\%) patients refused inclusion in the study; one $(1.5 \%)$ patient had cardiogenic pulmonary oedema secondary to chronic renal failure, and was on chronic haemodialysis; and one $(1.5 \%)$ patient with COPD had hypercapnia secondary to excessive absorption of benzodiazepines.

In the study population (11 males), 10 patients were assigned to each group. The mean age of the patients was $74 \pm 8.4 \mathrm{yrs}$ (range 52-89 yrs). Eight patients $(40 \%)$ had APO and $12(60 \%)$ had acute exacerbation of COPD. No patient included in the study had signs of acute coronary ischaemia. On the admission chest radiograph no patient had evidence of pneumothorax or pneumonia. The time between admission to the ED and the decision by the ED physician to propose the inclusion in this protocol was $55.9 \pm 106$ min in the placebo group and $82.8 \pm 216 \mathrm{~min}$ in the active bi-level NPPV group. The time needed to begin the noninvasive ventilation after inclusion was similar in the two groups $(21 \pm 6.8 \mathrm{~min}$ in the placebo group, $24 \pm 15.5 \mathrm{~min}$ in the active group). The baseline characteristics of the two groups were similar. As shown in table 1 , at the time of inclusion all patients had moderate-to-severe dyspnoea when self-assessed and when assessed by the care team; all had pallor and/or cyanosis, and most had sweating or agitation. Table 2 shows that all patients were tachycardic and tachypnoeic; all needed $\mathrm{O}_{2}$ supplementation and all but four were acidotic. The initial vital signs and the blood gases of the two groups were similar.

\section{Evolution of patients under placebo noninvasive positive-pressure ventilation}

After a mean of $29 \pm 17$ min of placebo NPPV plus conventional medical treatment, all 10 patients had experienced a clinical deterioration with persistency of tachypnoea (initial respiratory rate $37.5 \pm 7.3$, final $37.7 \pm 3.6(\mathrm{NS})$ ) (fig. 1) and tachycardia (initial cardiac frequency 125.8 \pm 20.3 , final $136.8 \pm 21.6 \quad(\mathrm{p}<0.01))$, major dyspnoea and sweating, agitation in nine patients and cyanosis in six patients. This clinical

Table 1.-Baseline characteristics of the study patients at the time of randomisation in the emergency dept and causes of acute respiratory failure

\begin{tabular}{lcc}
\hline Characteristics & \multicolumn{2}{c}{ Patients receiving } \\
\cline { 2 - 3 } & Bi-level NPPV & Placebo \\
\hline Subjects n & 10 & 10 \\
Age yrs & $71 \pm 9$ & $76 \pm 7$ \\
M:F & $7: 3$ & $4: 6$ \\
History of previous intubation & 1 & 0 \\
History of respiratory disease & 8 & 5 \\
Smoking history & 8 & 4 \\
Admission diagnosis & $3(30)$ & $5(50)$ \\
APO & $7(70)$ & $5(50)$ \\
COPD & & \\
Clinical conditions & 5 & 7 \\
Symptoms <24 h & 5 & 3 \\
Symptoms $>24$ h & $22.5 \pm 30$ & $18.1 \pm 13$ \\
Duration of dyspnoea $h$ & $8.5 \pm 1.55$ & $8.3 \pm 1.71$ \\
VAS patient & $8 \pm 1.15$ & $8.1 \pm 1.91$ \\
VAS nurse & $7.95 \pm 1.6$ & $7.9 \pm 1.91$ \\
VAS investigator & 2 & 1 \\
Moderate dyspnoea & 8 & 9 \\
Major/severe dyspnoea & 10 & 10 \\
Palor/cyanosis & 9 & 4 \\
Sweating & 0 & 3 \\
Agitation & 9 & 9 \\
Abnormality on ECG & 10 & 10 \\
Abnormal radiograph & & \\
\hline
\end{tabular}

Data are presented as mean $\pm \mathrm{SD}, \mathrm{n}$ or $\mathrm{n}(\%)$, unless otherwise stated. APO: acute pulmonary oedema; COPD: chronic obstructive pulmonary disease; ECG: electrocardiogram; NPPV: noninvasive positive-pressure ventilation; VAS: visual analog scale (0: normal breathing-10: unbearable dyspnoea). 
Table 2. - Baseline vital signs and arterial blood gas results of the study patients at the time of randomisation

\begin{tabular}{|c|c|c|c|}
\hline \multirow[t]{2}{*}{ Characteristics } & \multicolumn{2}{|c|}{ Patients receiving } & \multirow[t]{2}{*}{ p-value } \\
\hline & Bi-level NPPV & Placebo & \\
\hline Subjects $n$ & 10 & 10 & \\
\hline Heart rate beats $\cdot \mathrm{min}^{-1}$ & $122.4 \pm 22(74-158)$ & $122 \pm 20(95-150)$ & NS \\
\hline Respiratory rate breaths $\cdot \min ^{-1}$ & $36.7 \pm 10.3(20-52)$ & $37.5 \pm 7.2(24-47)$ & NS \\
\hline Added $\mathrm{O}_{2} \mathrm{~L} \cdot \mathrm{min}^{-1}$ & $3.8 \pm 3.5(1-10)$ & $5.7 \pm 2.63(1-8)$ & NS \\
\hline Arterial pH & $7.28 \pm 0.08(7.1-7.39)$ & $7.24 \pm 0.11(7.08-7.43)$ & NS \\
\hline$P \mathrm{a}, \mathrm{CO}_{2} \mathrm{mmHg}$ & $56.3 \pm 16.5(35-93)$ & $59.9 \pm 19(45-102)$ & NS \\
\hline$P \mathrm{a}, \mathrm{O}_{2} \mathrm{mmHg}$ & $78.3 \pm 37.2(37-155)$ & $64.2 \pm 19.7(41-92)$ & NS \\
\hline $\mathrm{Sa}_{\mathrm{a}, \mathrm{O}_{2}} \%$ & $87.9 \pm 11.3(61-96.1)$ & $83.5 \pm 12.1(61-96.2)$ & NS \\
\hline$S_{\mathrm{p}, \mathrm{O}_{2}} \%$ & $89.1 \pm 8.3(71-98)$ & $79.7 \pm 7.2(50-96)$ & NS \\
\hline
\end{tabular}

Data are presented as mean $\pm \mathrm{SD}$ (range). NS: not significant; $\mathrm{O}_{2}$ : oxygen; $\mathrm{Pa}, \mathrm{CO}_{2}$ : carbon dioxide tension in arterial blood; $\mathrm{Pa}, \mathrm{O}_{2}$ : $\mathrm{Pa}_{\mathrm{a}} \mathrm{O}_{2}$ : oxygen tension in arterial blood; $\mathrm{Sa}_{\mathrm{a}} \mathrm{O}_{2}$ : arterial oxygen saturation; $\mathrm{S}_{\mathrm{p}, \mathrm{O}_{2}}$ : transcutaneous $S_{\mathrm{a}, \mathrm{O}_{2}}$ measured from a pulse oxymeter with a finger probe; NPPV: noninvasive positive-pressure ventilation. $1 \mathrm{mmHg}=0.133 \mathrm{kpA}$.

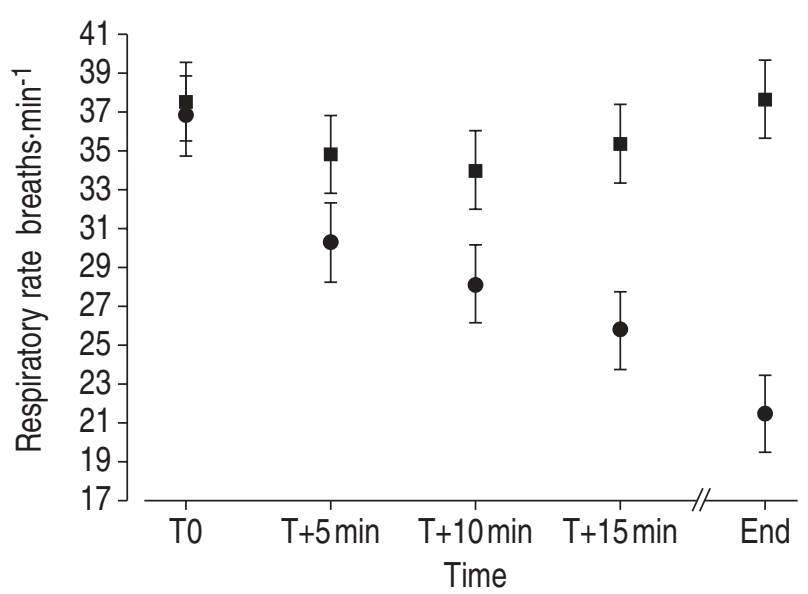

Fig. 1.-Evolution of the respiratory rate in the two groups of patients. "T0" refers to values at the time of inclusion. Note that the "End" time differs for both groups (see text for details). Oneway analysis of variance with repeated data showed no significant difference in the placebo group $(\bullet)$, and a significant decrease in the active noninvasive positive-pressure ventilation (NPPV) group (ש) $(\mathrm{p}<0.001)$.

deterioration was not paralleled by a worsening in arterial blood gases, which remained unchanged. The sternocleidomastoid EMG activity also remained unchanged or increased during this period (fig. 2). At this point in time, the attending physician recommended that all 10 patients be intubated and mechanically ventilated (table 3).

Evolution of patients under active noninvasive positivepressure ventilation

After a mean of $95 \pm 29$ min of active NPPV and conventional medical treatment (significantly longer than the placebo NPPV period), the attending physician recommended that NPPV could be safely discontinued in all 10 patients (table 3). Active NPPV

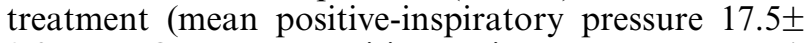
$2.9 \mathrm{cmH}_{2} \mathrm{O}$, mean positive-expiratory pressure $7 \pm$ $1.64 \mathrm{cmH}_{2} \mathrm{O}$ ) was characterised by a rapid clinical improvement, with decreases in respiratory rate (initial 36.8 \pm 11.7 , final $21.6 \pm 10.2(\mathrm{p}=0.01)$ ) (fig. 1) and cardiac frequency (initial 122.4 \pm 22.09 , final $105.9 \pm 27.5(\mathrm{p}=0.02))$, decreases in dyspnoea, and improvements in acidosis and hypercapnia (all statistically significant, see table 4). Upon application of NPPV the sternocleidomastoid EMG activity rapidly declined and remained at low levels throughout the rest of the treatment period.

Evolution of the placebo group under active noninvasive positive-pressure ventilation (rescue protocol)

The 10 patients failing placebo NPPV were put on active NPPV at the time when the decision to use intubation and mechanical ventilation by the attending physician was made. Seven of these 10 patients experienced a rapid clinical and blood-gases improvement, with decreases in respiratory rates (initial $37.7 \pm 3.6$, final $23.6 \pm 4.6(\mathrm{p}=0.005))$ and cardiac frequency (initial 136.8 \pm 21.6 , final $103.7 \pm 24(\mathrm{p}=0.005)$ ), dyspnoea sensation, acidosis and hypercapnia, and increases in $\mathrm{Pa}_{2} \mathrm{O}_{2}$ (all statistically significant, see table 4). The sternocleidomastoid EMG activity rapidly declined and remained at low values throughout the $63 \pm 34 \mathrm{~min}$ of duration of the rescue protocol (fig. 2).

Three patients failed the active NPPV rescue treatment and were finally intubated and mechanically ventilated (table 3 ). Two of these patients presented with haemodynamic shock after institution of the active bi-level NPPV. ECG showed abnormal leftventricular function $(<25 \%$ ECG estimated ejection fraction). Another patient was intubated as no signs of improvement with active bi-level NPPV were seen. A second chest radiograph revealed an asymmetry of the two hemithoraces. A computed tomography scan of the thorax showed an anterior-right pneumothorax.

\section{Patient outcomes}

No patient died in the first $24 \mathrm{~h}$ after admission. Three patients died afterwards (two in the active group and one in the placebo group). The causes of death in the active bi-level NPPV group were end-stage 
a)

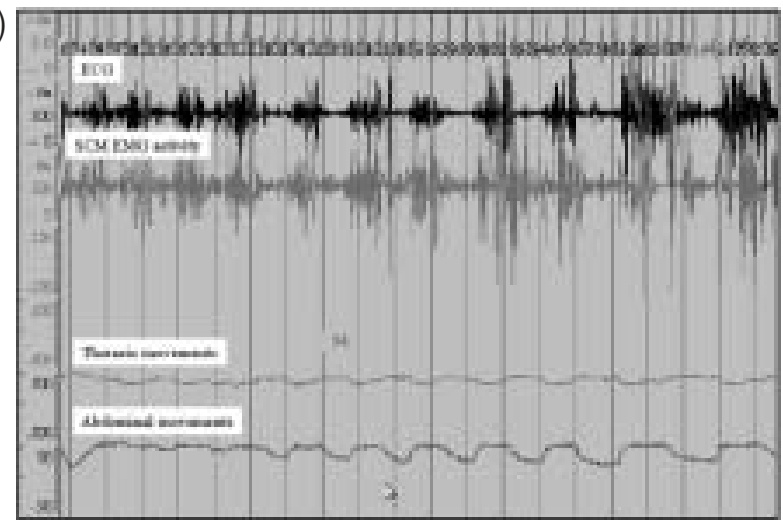

C)

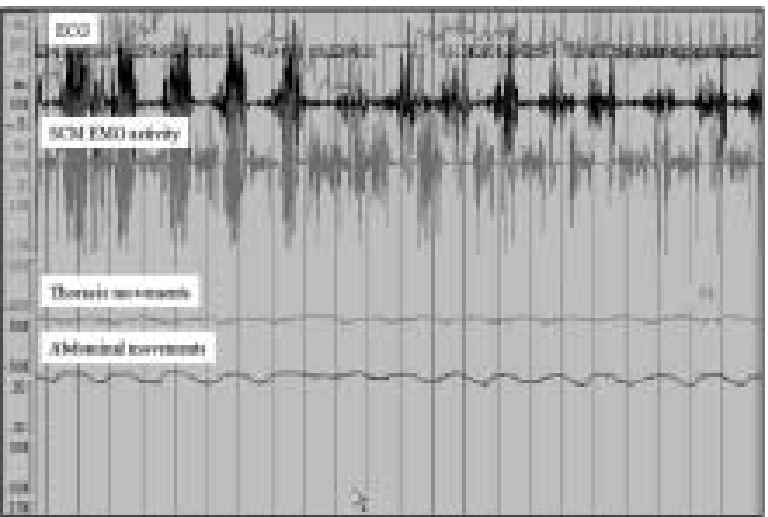

e)

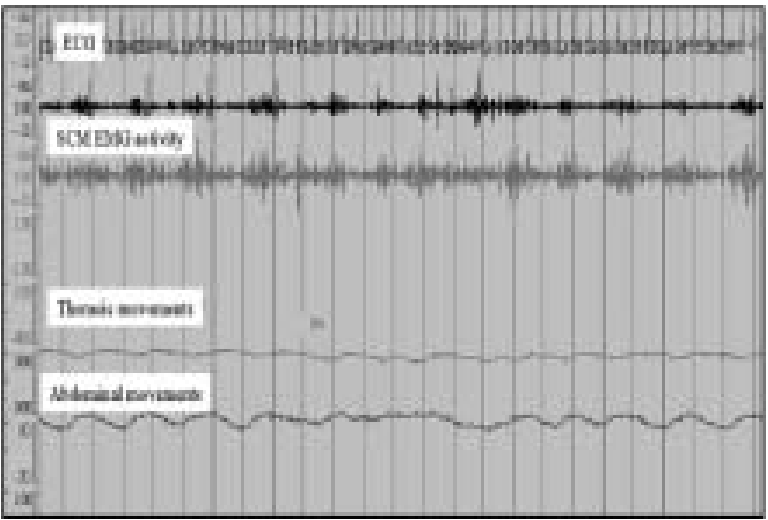

cardiac failure (after 3 days) and a haemorrhagic complication of a gastric cancer unknown at admission in a patient with COPD (after 6 days). In the placebo group, the cause of death was end-stage

Table 3. - Main patient outcomes

\begin{tabular}{lcc}
\hline Protocols & Success & Failure \\
\hline Active bi-level NPPV & 10 & 0 \\
Placebo NPPV & 0 & 10 \\
Rescue & 7 & 3
\end{tabular}

Failure: need of endotracheal intubation in the active bi-level noninvasive positive-pressure ventilation (NPPV) arm, and of crossing over to active NPPV (rescue) in the placebo arm. b)

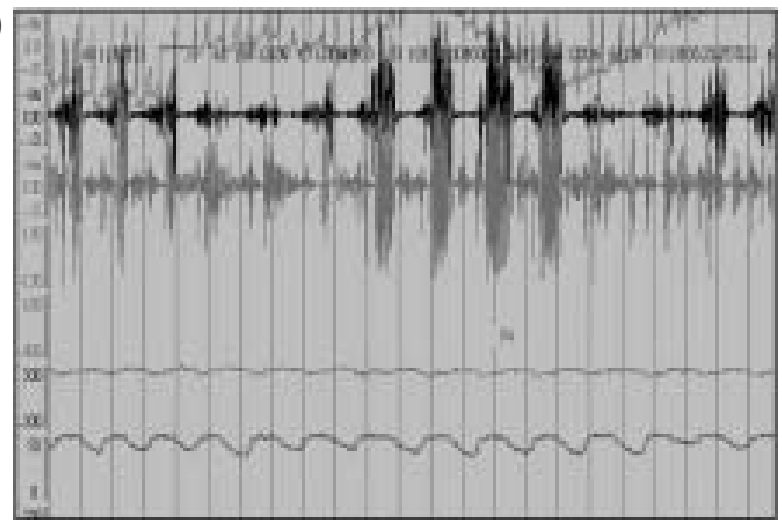

d)

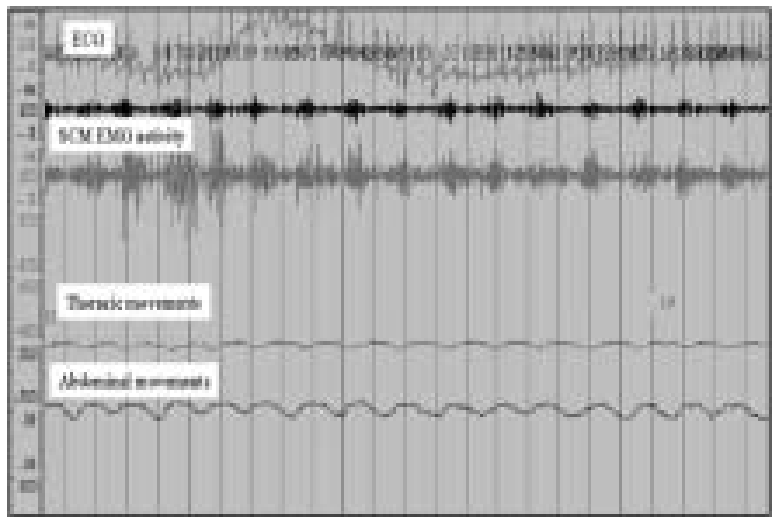

Fig. 2.-Evolution of the sternocleidomastoid (SCM) electromyogram (EMG) activity throughout the study period in one patient: a) conventional medical therapy; b) end of placebo noninvasive positive-pressure ventilation (NPPV); c) initiation of two-level NPPV; d) 1-min after bi-level NPPV; e) 11-min after bi-level NPPV. In each plot, the following signals are displayed from top to bottom: electrocardiogram (ECG); EMG of the SCM activity obtained from surface electrodes placed upon the left and right SCM muscles; and thoracic and abdominal movements obtained with a respiratory inductive plethysmograph. Note the prominent activity of SCM muscles with conventional medical therapy that persists at the end of placebo NPPV. Upon institution of the rescue protocol (active NPPV), note the decrease in the EMG signal amplitude.

cardiac failure. This patient was one of the two patients showing signs of cardiogenic shock upon institution of active NPPV (rescue protocol) after failing on placebo NPPV, and died on hospitalisation day 15. Five patients were discharged from the ED to the wards. Fifteen patients were admitted to the ICU after discharge from the ED (eight in the placebo group and seven in the active bi-level NPPV group). Ten of these patients received further treatment with NPPV in the ICU (three in the active and six in the placebo groups) for an average of $20 \mathrm{~h}$ during the first day in ICU. There was no difference in the ED length of stay (active NPPV $474 \pm 438 \mathrm{~min}$, placebo NPPV $309 \pm 184 \mathrm{~min}$ ) or in the total hospital length of stay (active NPPV 16 \pm 13.6 days, placebo NPPV 17.6 \pm 14.3 days), whereas there was a trend towards a greater 
ICU length of stay in the placebo group (active NPPV $2.7 \pm 3.5$ days, placebo NPPV 5.4 \pm 5.6 days).

\section{Work of breathing and end-tidal carbon dioxide}

The results of these experiments showed that the placebo NPPV device resulted in no change in the work of breathing or in $P \mathrm{ET}, \mathrm{CO}_{2}$, whereas the mask applied without the placebo NPPV led to an increase in $P$ ET, $\mathrm{CO}_{2}$ but no change in WOB. The mean values for WOB, expressed as WOB $\cdot \mathrm{L}$ of tidal volume ${ }^{-1}$ were as follows: spontaneous breathing $0.186 \pm 0.064$ Joules $(\mathrm{J}) \cdot \mathrm{L}^{-1}$, face mask $0.192 \pm 0.079 \mathrm{~J} \cdot \mathrm{L}^{-1}$, placebo NPPV $0.185 \pm 0.072 \mathrm{~J} \cdot \mathrm{L}^{-1}$, with no significant difference between these values as determined by the Wilcoxon test. The respective $P \mathrm{ET}, \mathrm{CO}_{2}$ values in $\mathrm{kPa}(\mathrm{mmHg})$ were: spontaneous breathing $5.0 \pm 0.4(37.82 \pm 2.99)$, face mask $4.5 \pm 0.4$ (34.04 \pm 2.92$)$, placebo NPPV $5.0 \pm 0.4 \quad(37.96 \pm 3) \quad(\mathrm{p}<0.03$ for face mask versus spontaneous breathing, $\mathrm{p}<0.02$ for face mask versus placebo NPPV, $\mathrm{p}=0.74$ for spontaneous breathing versus placebo NPPV, all determined by the Wilcoxon test).

\section{Discussion}

A small group of patients with severe respiratory failure, just before intubation and mechanical ventilation, have been studied. The application of active NPPV in the emergency department shortly after the arrival of these patients avoided the programmed intubation in all patients, and resulted in a rapid improvement of the patients' condition. This improvement was due to the application of ventilatory support and not due to the conventional medical treatment already instituted. The application of NPPV led to a true physiopathological result, and was not explained by a placebo effect. Placebo NPPV resulted in no change in the patients' clinical condition, that continued to worsen despite stabilisation of blood gases. Institution of active NPPV after failure of the placebo device (instead of actual intubation) led to an improvement in most patients. Active NPPV was not devoid of unwanted complications, but they were not lethal in this study. The differences between active and placebo NPPV were so great that the study was stopped after including one-third of the planned number of patients.

Previous studies on the usefulness of noninvasive assisted ventilation in a number of conditions leading to ARF have shown that this form of therapy can result in the avoidance of endotracheal intubation, reduction in the number of complications such as nosocomial infections, reductions in the length of stay of the patients in the ICU and sometimes in the hospital, and in some studies decreases in mortality [1-3]. In the last few years, NPPV has been applied to patients with acute exacerbation of COPD [4-8, 19], status asthmaticus [20, 21], community acquired pneumonia [11], acute pulmonary oedema $[9,10,22]$, ARF after solid-organ transplantation, and ARF in haematological malignancies or immunosuppressed 
patients [23-26]. There are enough data suggesting that NPPV represents a useful added value equal to and above that of conventional medical treatment. However, at least one study has shown that the addition of NPPV to conventional medical treatment resulted in no change in the outcome of the patients, suggesting that either in less-severe patients NPPV is not necessary and medical treatment suffices to manage the condition, or that NPPV is a placebo treatment without real effect [12]. The problem of controlling for a placebo-effect treatment is pervasive, and it has been suggested that the possibility of a powerful placebo effect is much higher for treatment with machines than with medical treatment, where it is already significant [27-29]. Most studies performed to date with NPPV were randomised but not placebo controlled. One might suppose that placebo effects should be small or even nonexistent in the context of severe diseases or life threatening conditions. However this remains to be proven, since practically no data are available on placebo effects in severe conditions. One could even hypothesise that NPPV, by its very cumbersome nature, renders sleep (and its related deterioration of ventilation) almost impossible. Thus, NPPV could act simply by avoiding the sleep-related deterioration of hypercapnia and hypoxia. Therefore, even through one could a priori dismiss the possibility of placebo effects in emergency conditions there is still the need to prove that placebo effects indeed do not exist. The present data are the first to show that there is no placebo effect in NPPV applied in patients with acute severe, life threatening, respiratory failure.

No difference in outcome (intubation, mortality, length of stay) between the two groups of patients was found. This is exactly what was to be expected if NPPV had no placebo effect. Indeed, it should be remembered that all patients were considered for intubation at the time of inclusion. None were intubated in the active NPPV arm. In the placebo group, the decision to intubate was not modified after a 30-min lapse. The fact that these patients were not intubated but submitted to the rescue (active NPPV) protocol does not change the decision of the attending physician: placebo NPPV had failed, and intubation was to be applied. However, since the institution of the rescue protocol resulted in the avoidance of actual intubation in seven out of 10 patients, and since it is actual intubation (and its complications) which explain the differences in outcomes previously demonstrated between intubation and active NPPV, it follows that no such difference was to be expected in this study. The total failure rate of active NPPV in the present study was $15 \%$, a figure in the range of the published studies in patients with a similar degree of severity (table 5) [7, 10,12,13,15,16, 19, 30-35]. The fact that the attending physician was not blinded to the active or placebo nature of the treatment under study could have influenced the results. Indeed, the physician, knowing that no active treatment was being applied to the patient in severe ARF, could be quick to declare the treatment as a failure so that the active treatment be applied as soon as possible. Had the physician been blind to the nature of the treatment there was the possibility that the medical treatment

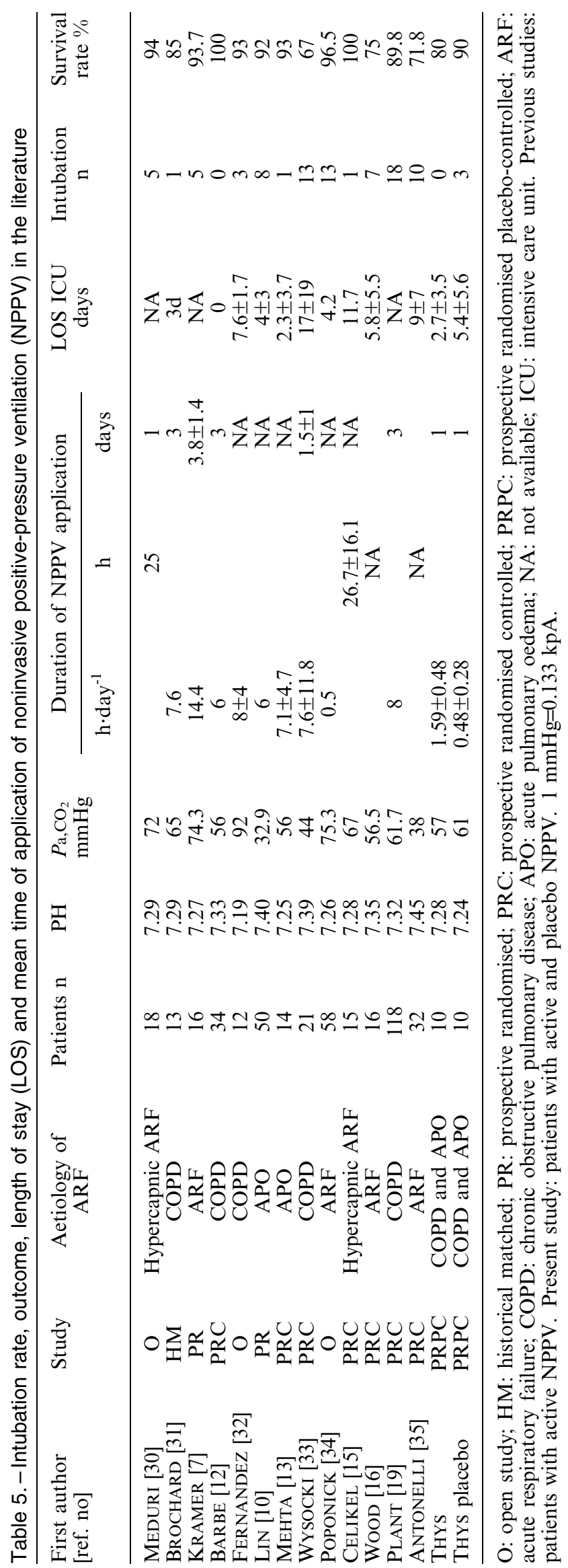


may have been given for a longer period and would have eventually relieved the patient. The authors believe that this caveat, due to the single-blinded type of study performed (it would have been quite difficult to conceal the mask and connector from the attending physician) should not be considered as a major weakness. Indeed, the speed of clinical improvement in the active group was such that even if the physician had been blinded to the treatment, he could not have been blinded to the respiratory rate (fig. 1).

One might be surprised by the $100 \%$ failure rate of the sham NPPV group of patients. Indeed, in the published controlled studies, some patients did improve under medical therapy $[5,7,12]$. However, it should be recalled that the patients were included in the study only after a period of medical treatment had been administered, and the patient had failed to improve, so that intubation was already considered at inclusion.

All patients in the placebo group showed a rapid clinical deterioration. This was not due to the application of a placebo NPPV. Indeed, it had been verified experimentally in normal subjects that the placebo device had no detrimental effect on WOB and did not favour hypercapnia through its added dead space. This was also confirmed by the absence of deterioration in blood gases despite the clinical worsening, and by the absence of untoward haemodynamic effects, that were rapidly apparent with active NPPV in two of the patients. One further patient, who was in the placebo-treatment group and was also included in the rescue-active treatment group after failure of the placebo protocol, was found to have a pneumothorax. It is not certain whether the pneumothorax was already present but not visible on the conventional chest radiograph before instituting NPPV, or whether it was a complication of the treatment. This patient had to be intubated and mechanically ventilated, but survived the acute disease and left the hospital after 15 days. Thus, the three patients needing intubation failed NPPV for clear medical reasons and not due to a delay in medical attention explained by the experimental protocol. In fact, the safety of the patients was a primary concern, and the attending physician was present throughout the whole protocol in all patients. Other frequently described complications (skin damage, gastric dilatation) were not seen in this study, perhaps because of the short time of NPPV treatment. This could be related to the very early nature of noninvasive ventilation, as suggested by CELIKeL et al. [15], although this remains to be satisfactorily proven.

When the study was initiated, the need for inclusion was evaluated at 60 patients. The significant difference between the two groups led to the decision to interrupt the study after the inclusion of 20 patients. Due to the small number of patients at study interruption, it was difficult to draw statistically valid conclusions when analysing the patients by diagnostic category, i.e. COPD on the one hand and APO on the other hand. Nevertheless, the general picture of failure of placebo NPPV and success of the active treatment was true in both groups of patients (table 6).

Although it was not the main outcome of interest in this study, it was found that the EMG signal of the sternocleidomastoid muscle was extremely helpful to rapidly follow the evolution of the patients: in all cases of NPPV success, a rapid and clear-cut decrease in EMG activity was observed, whereas there was no such decrease in patients with placebo NPPV or in patients with failure on active NPPV. The EMG signal can be obtained easily at the bedside, it is noninvasive, and might represent a useful contribution in monitoring patients under NPPV. PoPONICK et al. [34] had suggested that patients not improving after $30 \mathrm{~min}$ of NPPV needed endotracheal intubation. Interestingly, this was the exact average time of application of placebo NPPV in the present study.

Table 6. - Arterial blood-gas evolution in the study patients by pathology

\begin{tabular}{|c|c|c|c|c|c|c|}
\hline & \multicolumn{3}{|c|}{ Placebo group } & \multicolumn{3}{|c|}{ Bi-level NPPV group } \\
\hline & Before & After & $\mathrm{p}$-value & Before & After & $\mathrm{p}$-value \\
\hline \multicolumn{7}{|l|}{ APO } \\
\hline Added $\mathrm{O}_{2} \mathrm{~L} \cdot \mathrm{min}^{-1}$ & $7.4 \pm 0.89(6-8)$ & $8.2 \pm 1.48(6-10)$ & NS & $6.6 \pm 5.77(1-10)$ & $5.6 \pm 2.08(4-8)$ & NA \\
\hline Arterial pH & $7.24 \pm 0.15(7.08-7.43)$ & $7.28 \pm 0.16(7.08-7.48)$ & NS & $7.25 \pm 0.1(7.1-7.36)$ & $7.32 \pm 0.02(7.3-7.35)$ & NA \\
\hline$P \mathrm{a}, \mathrm{CO}_{2} \mathrm{mmHg}$ & $51 \pm 8.3(45-65)$ & $48.6 \pm 5.7(41-56)$ & NS & $50 \pm 7.5(45-61)$ & $37 \pm 6.9(29-47)$ & NA \\
\hline$P \mathrm{a}, \mathrm{O}_{2} \mathrm{mmHg}$ & $62 \pm 18.6(43-89)$ & $54 \pm 9.4(43-63)$ & NS & $89 \pm 44(37-155)$ & $89 \pm 20(60-120)$ & NA \\
\hline $\mathrm{Sa}_{\mathrm{a}, \mathrm{O}_{2}} \%$ & $83.6 \pm 10.9(67.6-96.6)$ & $81 \pm 11.2(67.6-91.7)$ & NS & $84.4 \pm 18.1(64.1-99.1)$ & $94.4 \pm 5.1(88.6-98.2)$ & NA \\
\hline $\mathrm{Sp}, \mathrm{O}_{2} \%$ & $75.4 \pm 20.1(50-96)$ & $77.2 \pm 17.8(61-96)$ & NS & $90.6 \pm 8.73(81-98)$ & $91.6 \pm 11.8(78-99)$ & NA \\
\hline \multicolumn{7}{|l|}{ COPD } \\
\hline Added $\mathrm{O}_{2} \mathrm{~L} \cdot \mathrm{min}^{-1}$ & $4 \pm 2.74(1-8)$ & $3.2 \pm 2.9(1-8)$ & NS & $2.6 \pm 1.3(1-3.5)$ & $1.1 \pm 0.9(0-2)$ & NS \\
\hline Arterial pH & $7.24 \pm 0.09(7.13-7.32)$ & $7.24 \pm 0.05(7.17-7.32)$ & NS & $7.30 \pm 0.05(7.22-7.39)$ & $7.38 \pm 0.05(7.3-7.47)$ & 0.002 \\
\hline$P \mathrm{a}, \mathrm{CO}_{2} \mathrm{mmHg}$ & $68.8 \pm 23.5(46-102)$ & $64 \pm 26.5(40-107)$ & NS & $59.1 \pm 18.5(35-9)$ & $48 \pm 13.4(32-65)$ & 0.03 \\
\hline $\mathrm{Pa}_{\mathrm{a}} \mathrm{O}_{2} \mathrm{mmHg}$ & $66.2 \pm 22.7(41-92)$ & $73.6 \pm 38(35-129)$ & NS & $73.4 \pm 28(41-127)$ & $68.7 \pm 21.8(45-102)$ & NS \\
\hline $\mathrm{S}_{\mathrm{a}, \mathrm{O}_{2}} \%$ & $83.5 \pm 14.5(60.2-96.4)$ & $81.4 \pm 19.4(53.3-98.1)$ & NS & $89.5 \pm 8.5(72.1-98.3)$ & $90.4 \pm 6.6(79.6-97.3)$ & NS \\
\hline$S_{\mathrm{p}, \mathrm{O}_{2}} \%$ & $84 \pm 14.7(58-92)$ & $83.2 \pm 18.7(50-94)$ & NS & $88.4 \pm 8.8(71-97)$ & $91.3 \pm 3.8(87-97)$ & NS \\
\hline
\end{tabular}

Data are presented as mean \pm SD (range). NS: not significant; NA: not applicable; APO: acute pulmonary oedema; COPD: chronic obstructive pulmonary disease; $\mathrm{O}_{2}$ : oxygen; $\mathrm{P}_{\mathrm{a}}, \mathrm{CO}_{2}$ : carbon dioxide tension in arterial blood; $P \mathrm{a}, \mathrm{O}_{2}$ : oxygen tension in arterial blood; $\mathrm{Sa}_{\mathrm{a}} \mathrm{O}_{2}$ : arterial oxygen saturation; $\mathrm{Sp}_{\mathrm{p}} \mathrm{O}_{2}$ : transcutaneous $\mathrm{Sa}_{\mathrm{a}} \mathrm{O}_{2}$ measured from a pulse oxymeter with a finger probe. APO: placebo group $n=5$; bi-level NPPV group $n=3$; COPD: placebo group $n=5$; bi-level NPPV group $\mathrm{n}=7.1 \mathrm{mmHg}=0.133 \mathrm{kpA}$. 


\section{Conclusion}

To conclude, this prospective, randomised placebocontrolled study is the first to compare, beyond-standard medical therapy, noninvasive positive-pressure ventilation with a similar placebo device. Early application of bi-level noninvasive positive-pressure ventilation to patients with acute respiratory failure due to acute exacerbation of chronic obstructive pulmonary disease or acute pulmonary oedema leads to a rapid improvement in clinical status and blood gases, that differs substantially from the evolution of similar patients treated with conventional medical therapy and a placebo noninvasive positive-pressure ventilation device.

\begin{abstract}
Acknowledgements. The authors would like to thank C. Veriter, F. Verschuren, O. Dozin, J-B. Michotte, M.P. Matte for their help in collecting the data. The authors would also like to thank A. El Gariani, A. Elamly, P. Janssens, E. Marion, P. Meert, the nursing staff of the emergency department, R. Kessler, P-F. Laterre, W. D'Hoore, M. Blondiau, E. Choppin, I. Coca, C. Decnud, P. Deprez, S. Deroock, G. Deschietere, M. Schiffers, A. Hendricks, C. Lacroix, S. Redant and N. Stroobant for their continuous support and help during this study. The main author thanks A. Piron for his perpetual support.
\end{abstract}

\section{References}

1. Pingleton SK. Complications of acute respiratory failure. Am Rev Respir Dis 1988; 137: 1463-1493.

2. Colice GL, Stukel TA, Dain B. Laryngeal complications of prolonged intubation. Chest 1989; 96: 877-884.

3. Craven DE, Steger KA. Epidemiology of nosocomial pneumonia. New perspectives on an old disease. Chest 1995; 108: Suppl. 2, 1S-16S.

4. Ahmed AH, Fenwick L, Angus RM, Peacock AJ. Nasal ventilation versus doxapram in the treatment of type II respiratory failure complicating chronic airflow obstruction (abstract). Thorax 1992; 1: 858.

5. Bott J, Carroll MP, Conway JH, et al. Randomised controlled trial of nasal ventilation in acute ventilatory failure due to chronic obstructive airways disease. Lancet 1993; 341: 1555-1557.

6. Brochard L, Mancebo J, Wysocki M, et al. Noninvasive ventilation for acute exacerbations of chronic obstructive pulmonary disease. $N$ Engl J Med 1995; 333: 817822.

7. Kramer N, Meyer TJ, Meharg J, Cece RD, Hill NS. Randomized, prospective trial of noninvasive positive pressure ventilation in acute respiratory failure. $\mathrm{Am}$ J Respir Crit Care Med 1995; 151: 1799-1806.

8. Daskalopoulou E, Tsara V, Fekete K, Koutsantas V, Christaki $\mathrm{P}$. Treatment of acute respiratory failure in COPD patients with positive airway pressure via nasal mask (NPPV) (abstract). Chest 1993; 103: 271S.

9. Bersten AD, Holt AW, Vedig AE, Skowronski GA, Baggoley CJ. Treatment of severe cardiogenic pulmonary edema with continuous positive airway pressure delivered by face mask. N Engl J Med 1991; 325: 1825 1830.

10. Lin M, Yang YF, Chiang HT, Chang MS, Chiang BN, Cheitlin MD. Reappraisal of continuous positive airway pressure therapy in acute cardiogenic pulmonary edema. Short-term results and long-term followup. Chest 1995; 107: 1379-1386.

11. Confalonieri M, Potena A, Carbone G, Porta RD, Tolley EA, Umberto MG. Acute respiratory failure in patients with severe community-acquired pneumonia. A prospective randomized evaluation of noninvasive ventilation. Am J Respir Crit Care Med 1999; 160: $1585-1591$.

12. Barbe F, Togores B, Rubi M, Pons S, Maimo A, Agusti AG. Noninvasive ventilatory support does not facilitate recovery from acute respiratory failure in chronic obstructive pulmonary disease. Eur Respir $J$ 1996; 9: 1240-1245.

13. Mehta S, Jay GD, Woolard RH, et al. Randomized, prospective trial of bilevel versus continuous positive airway pressure in acute pulmonary edema. Crit Care Med 1997; 25: 620-628.

14. Pollack C, Torres MT, Alexander L. Feasibility study of the use of bilevel positive airway pressure for respiratory support in the emergency department. Ann Emerg Med 1996; 27: 189-192.

15. Celikel T, Sungur M, Ceyhan B, Karakurt S. Comparison of noninvasive positive pressure ventilation with standard medical therapy in hypercapnic acute respiratory failure. Chest 1998; 114: 1636-1642.

16. Wood KA, Lewis L, Von Harz B, Kollef MH. The use of noninvasive positive pressure ventilation in the emergency department: results of a randomized clinical trial. Chest 1998; 113: 1339-1346.

17. Thys F, Roeseler J, Delaere S, et al. Two-level noninvasive positive pressure ventilation in the initial treatment of acute respiratory failure in an emergency department. Eur J Emerg Med 1999; 6: 207-214.

18. Clark CJ, Downie CC. A method for the rapid determination of the number of patients to include in a controlled clinical trial. Lancet 1966; 2: 13571358.

19. Plant PK, Owen JL, Elliott MW. Early use of noninvasive ventilation for acute exacerbations of chronic obstructive pulmonary disease on general respiratory wards: a multicentre randomised controlled trial. Lancet 2000; 355: 1931-1935.

20. Meduri GU, Cook TR, Turner RE, Cohen M, Leeper KV. Noninvasive positive pressure ventilation in status asthmaticus. Chest 1996; 110: 767-774.

21. Thys F, Roeseler J, Marion E, et al. Non invasive ventilation in severe status asthmaticus, a new therapeutic approach? Two case reports. Réan Urg 1998; 7: 423-426.

22. Masip J, Betbese AJ, Paez J, et al. Non-invasive pressure support ventilation versus conventional oxygen therapy in the acute cardiogenic pulmonary oedema : a randomised trial. Lancet 2000; 356: $2126-$ 2132.

23. Conti G, Spadetta G, Rocco M, et al. Non invasive ventilation in the treatment of FUdr-induced lesional pulmonary oedema. Minerva Anestesiol 2000; 66: 561564.

24. Grant RK, Boots RJ, McEvoy JD. Bilevel noninvasive ventilation in malignant large airways obstruction during chemotherapy and radiotherapy. Anaesth Intensive Care 1999; 27: 295-297. 
25. Rabitsch W, Staudinger T, Brugger SA, et al. Successful management of adult respiratory distress syndrome (ARDS) after high-dose chemotherapy and peripheral blood progenitor cell rescue by non-invasive ventilatory support. Bone Marrow Transplant 1998; 21: 1067-1069.

26. Hilbert G, Gruson D, Vargas F, et al. Noninvasive ventilation in immunosuppressed patients with pulmonary infiltrates, fever, and acute respiratory failure. $N$ Engl J Med 2001; 15: 481-487.

27. Wright J, Johns R, Watt I, Melville A, Sheldon T. Health effects of obstructive sleep apnoea and the effectiveness of continuous positive airways pressure: a systematic review of the research evidence. $B M J$ 1997; 314: 851-860.

28. Wright J, Sheldon T. The efficacy of nasal continuous positive airway pressure in the treatment of obstructive sleep apnea syndrome is not proven (editorial). Am J Respir Crit Care Med 2000; 161: 1776-1778.

29. Davies RJ, Stradling JR. The efficacy of nasal continuous positive airway pressure in the treatment of obstructive sleep apnea syndrome is proven (editorial). Am J Respir Crit Care Med 2000; 161: 1775-1776.

30. Meduri GU, Abou SN, Fox RC, Jones CB, Leeper
KV, Wunderink RG. Noninvasive face mask mechanical ventilation in patients with acute hypercapnic respiratory failure. Chest 1991; 100: 445-454.

31. Brochard L, Isabey $\mathrm{D}$, Piquet $\mathrm{J}$, et al. Reversal of acute exacerbations of chronic obstructive lung disease by inspiratory assistance with a face mask. $N$ Engl J Med 1990; 323: 1523-1530.

32. Fernandez R, Blanch L, Valles J, Baigorri F, Artigas A. Pressure support ventilation via face mask in acute respiratory failure in hypercapnic COPD patients. Intensive Care Med 1993; 19: 456-461.

33. Wysocki M, Tric L, Wolff MA, Millet H, Herman B. Noninvasive pressure support ventilation in patients with acute respiratory failure. A randomized comparison with conventional therapy. Chest 1995; 107: 761768.

34. Poponick JM, Renston JP, Bennett RP, Emerman CL. Use of a ventilatory support system (BiPAP) for acute respiratory failure in the emergency department. Chest 1999; 116: 166-171.

35. Antonelli M, Conti G, Rocco M, et al. A comparison of noninvasive positive-pressure ventilation and conventional mechanical ventilation in patients with acute respiratory failure. N Engl J Med 1998; 339: 429-435. 